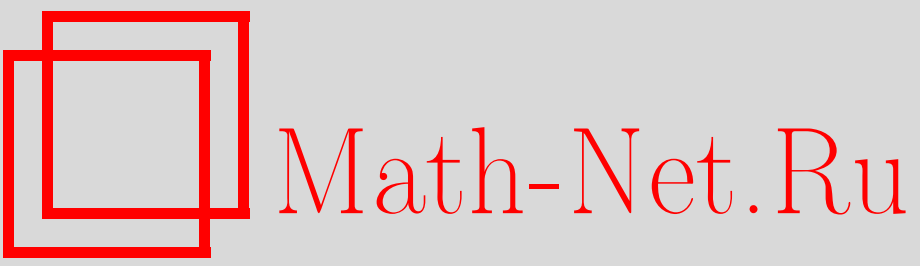

В. И. Козориз, Ю. Р. Мусин, К задаче рассеяния классической частицы со спином в кулоновском поле, ТМ $\Phi, 2004$, том 138, номер 2, 338-348

DOI: https://doi.org/10.4213/tmf23

Использование Общероссийского математического портала Math-Net.Ru подразумевает, что вы прочитали и согласны с пользовательским соглашением

http://www . mathnet.ru/rus/agreement

Параметры загрузки:

IP : 54.92 .164 .108

26 апреля 2023 г., 18:32:23 


\author{
ТЕОРЕТИЧЕСКАЯ \\ И МАТЕМАТИЧЕСКАЯ \\ ФИЗИКА \\ Том 138, № 2 \\ февраль, 2004
}

(C) $\mathbf{2 0 0 4}$ г.

В.И. Козориз*, Ю.Р. Мусин*

\title{
К ЗАДАЧЕ РАССЕЯНИЯ КЛАССИЧЕСКОЙ ЧАСТИЦЫ СО СПИНОМ В КУЛОНОВСКОМ ПОЛЕ
}

Рассмотрена задача рассеяния релятивистского электрона со спином в кулоновском поле в рамках псевдоклассической механики. Получено аналитическое выражение для угла рассеяния и даны предельные оценки параметров рассеяния для бесспинового и нерелятивистского случаев. Для случая малых углов рассматриваемая псевдоклассическая модель приводит к известной квантово-механической формуле Мотта.

Ключевые слова: псевдоклассическая механика, суперсимметрия, суперчастица, уравнения Баргмана-Мишеля-Телегди, формула Мотта, рассеяние.

\section{1. ВВЕДЕНИЕ}

Задача об упругом рассеянии электрона в кулоновском поле подробно изучена как на классическом, так и на квантовом уровне [1]-[6]. Тем не менее остался неисследованным вопрос о вкладе в сечения рассеяния чисто квантовых эффектов (см. таблицу). Как известно, формула Резерфорда, отвечающая случаю рассеяния электрона без учета спина, может быть получена как в классической, так и в квантовой теории [1]-[4]. Формула Мотта, описывающая сечение рассеяния с учетом спина электрона, справедлива как в релятивистской, так и в нерелятивистской квантовой механике [5]. Отсутствие классических моделей спина до недавнего времени делало невозможным прямое сравнение квантово-механических и классических подходов. С созданием псевдоклассической механики (механики над алгеброй Грассмана [6], [7]) и построением на ее основе суперсимметричных моделей электрона с учетом спина [8], [9] это стало возможным. В рамках нерелятивистской псевдоклассической механики задача о движении частицы со спином в центральном поле была решена Березиным и Мариновым [6], но соответствующее сечение рассеяния в кулоновском поле они не рассчитывали. В работе [10] было найдено аналитическое решение задачи о движении релятивистского суперсимметричного электрона в кулоновском поле. В данной работе мы находим угол рассеяния и сечение рассеяния для этой псевдомеханической модели.

* Московский авиационный институт им. С. Орджоникидзе, Москва, Россия. E-mail: vkozoriz@rambler.ru; maino@mai.ru 
Исследование движения электрона в кулоновском поле

\begin{tabular}{|c|c|c|c|c|}
\hline & \multicolumn{2}{|l|}{ Теории } & \multicolumn{2}{|c|}{ Частицы } \\
\hline & & & $\begin{array}{c}\text { Бесспиновая } \\
\text { частица }\end{array}$ & $\begin{array}{l}\text { Частища со } \\
\text { спином } 1 / 2\end{array}$ \\
\hline \multirow{4}{*}{$\begin{array}{c}\text { Классическая } \\
\text { механика }\end{array}$} & \multirow{2}{*}{$\begin{array}{c}\text { Нереляти- } \\
\text { вистская }\end{array}$} & \begin{tabular}{|l} 
Уравнения \\
движения \\
\end{tabular} & Ньютон & $\begin{array}{c}\text { Псевдоклассическая } \\
\text { механика, Березин [6] }\end{array}$ \\
\hline & & $\begin{array}{c}\text { Сечение } \\
\text { рассеяния }\end{array}$ & Резерфорд [1] & не получено \\
\hline & \multirow{2}{*}{$\begin{array}{l}\text { Реляти- } \\
\text { вистская }\end{array}$} & $\begin{array}{l}\text { Уравнения } \\
\text { движения }\end{array}$ & CTO & $\begin{array}{c}\text { Псевдоклассическая } \\
\text { механика, Баргман } \\
\text { Мишель, Телегди }\end{array}$ \\
\hline & & $\begin{array}{c}\text { Сечение } \\
\text { рассеяния }\end{array}$ & Резерфорд [2] & Данная статья \\
\hline \multirow{4}{*}{$\begin{array}{c}\text { Квантовая } \\
\text { механика }\end{array}$} & \multirow{2}{*}{$\begin{array}{c}\text { Нереляти- } \\
\text { вистская }\end{array}$} & $\begin{array}{l}\text { Уравнения } \\
\text { движения }\end{array}$ & Шредингер & Паули \\
\hline & & $\begin{array}{c}\text { Сечение } \\
\text { рассеяния }\end{array}$ & Резерфорд [3] & Мотт [5] \\
\hline & \multirow{2}{*}{$\begin{array}{l}\text { Реляти- } \\
\text { вистская }\end{array}$} & $\begin{array}{l}\text { Уравнения } \\
\text { движения } \\
\end{array}$ & Клейн, Гордон & Дирак \\
\hline & & $\begin{array}{c}\text { Сечение } \\
\text { рассеяния }\end{array}$ & Резерфорд [4] & Мотт [5] \\
\hline
\end{tabular}

\section{2. ОПИСАНИЕ МОДЕЛИ}

В качестве псевдоклассической модели электрона мы используем вариант релятивистской модели Ди Векиа-Равндела [6] с фиксированной калибровкой. В основе модели лежит процедура суперрасширения собственного времени частишы $s$ до супервремени $(s, \tau)$. Дополнительная временна́я координата $\tau$ является антикоммутирующей, а сами переменные $s$ и $\tau$ интерпретируются как четная и нечетная образующие алгебры Грассмана $G_{1,1}[9]$ со следуюшими свойствами:

$$
[s, s]=0, \quad\{\tau, \tau\}=0, \quad[s, \tau]=0,
$$

где $[\cdot, \cdot \cdot]$ - коммутатор, a $\{\cdot, \cdot\}$ - антикоммутатор.

Геометрия плоского супервремени задается дифференциальной формой

$$
\omega=d s-i \tau d \tau
$$

Она инвариантна относительно трансляций вдоль четного времени

$$
s^{\prime}=s+\tilde{\alpha}, \quad \tau^{\prime}=\tau,
$$


где $\tilde{\alpha}-$ произвольная константа, и суперповоротов

$$
s^{\prime}=s+i \epsilon \tau, \quad \tau^{\prime}=\tau+\epsilon,
$$

где $\epsilon$ - произвольная нечетная константа алгебры Грассмана.

Векторные поля на супервремени называются суперкоординатами. Связь между суперкоординатой $X^{\mu}(s, \tau)$ и обычными координатами устанавливается разложением суперкоординаты по нечетному времени. В силу нильпотентности последнего разложение обрывается на втором члене:

$$
X^{\mu}(s, \tau)=x^{\mu}(s)+i \theta^{\mu}(s) \tau, \quad \mu=0,1,2,3
$$

где $x^{\mu}(s)$ - пространственные координаты, а $\theta^{\mu}(s)$ интерпретируются как внутренние (спиновые) координаты, причем

$$
i \theta^{\mu}(s)=\left.\frac{\partial X^{\mu}}{\partial \tau}\right|_{\tau=0}
$$

Генераторы преобразований суперкоординат $X^{\mu}(s, \tau)$, порождаемые движениями плоскости $(s, \tau)$, имеют следующий вид:

а) трансляция:

$$
\widetilde{H}=i \frac{\partial}{\partial s}, \quad \delta X^{\mu}=-\tilde{\alpha} i \widetilde{H} X^{\mu}
$$

б) суперповорот:

$$
Q=i \tau \frac{\partial}{\partial s}+\frac{\partial}{\partial \tau}, \quad \delta X^{\mu}=\epsilon Q X^{\mu}
$$

Операторы $\widetilde{H}, Q$ и сопряженный к $Q$ оператор

$$
D=\bar{Q}=-i \tau \frac{\partial}{\partial s}+\frac{\partial}{\partial \tau}
$$

образуют два представления алгебры суперсимметрии (SUSY)

$$
\begin{array}{ll}
(Q, \widetilde{H}): \quad[Q, \widetilde{H}]=[\widetilde{H}, \widetilde{H}]=0, & \{Q, Q\}=2 \widetilde{H} \\
(D, \widetilde{H}):[D, \widetilde{H}]=[\widetilde{H}, \widetilde{H}]=0, & \{D, D\}=-2 \widetilde{H}
\end{array}
$$

Действие для свободной частицы со спином имеет вид [8]

$$
S=\frac{1}{2} \int d s \int d \tau D X^{\mu} D D X_{\mu} .
$$


Лагранжев множитель выберем равным единице. Считаем для простоты, что $c=m=1$, где $c$ - скорость света, $m$ - масса частицы. После подстановки выражений для $D$ и $X^{\mu}$ получим выражение

$$
S=\frac{1}{2} \int d s\left(\dot{x}_{\mu} \dot{x}^{\mu}+i \theta_{\mu} \dot{\theta}^{\mu}\right)
$$

где точкой обозначается производная по собственному времени $s$.

Для введения взаимодействия "удлиним" производную:

$$
D D X^{\mu} \rightarrow D D X^{\mu}+2 i e A^{\mu}(X), \quad X=\left(X^{0}, X^{1}, X^{2}, X^{3}\right),
$$

где $A^{\mu}(X)$ - 4-потенциал электромагнитного поля, его можно представить в виде

$$
A^{\mu}(X)=A^{\mu}(x)+\left.\frac{\partial A^{\mu}(x)}{\partial X^{\nu}}\right|_{\tau=0} i \theta^{\nu} \tau, \quad x=\left(x^{0}, x^{1}, x^{2}, x^{3}\right) .
$$

Подставляя в исходное действие (3) новую производную и выражения для $A^{\mu}$ и $X^{\mu}$, получим следуюшее выражение:

$$
S=\int d s\left(\frac{1}{2} \dot{x}^{\mu} \dot{x}_{\mu}+\frac{1}{2} i \theta^{\mu} \dot{\theta}_{\mu}+q \dot{x}^{\mu} A_{\mu}+i q \partial_{\nu} A_{\mu} \theta^{\mu} \theta^{\nu}\right)
$$

Уравнения движения, вытекающие из этого действия, имеют вид

$$
\begin{aligned}
\ddot{x}_{\mu}-q F_{\mu \nu} \dot{x}^{\nu}-\frac{q}{2} S^{\nu \alpha} \partial_{\mu} F_{\nu \alpha} & =0, \\
\dot{\theta}_{\mu}-q F_{\mu \nu} \theta^{\nu} & =0,
\end{aligned}
$$

где $S^{\nu \alpha}=-i \theta^{\nu} \theta^{\alpha}-$ тензор спина, $F_{\mu \nu}=\partial_{\mu} A_{\nu}-\partial_{\nu} A_{\mu}$ - тензор электромагнитного поля. Уравнения (4) формально совпадают с традиционными уравнениями БаргманаМишеля-Телегди (БМТ) для частицы с гиромагнитным отношением $g=2$ (следствие суперсимметрии (3)). Однако уравнения (4) являются только грассмановым аналогом уравнений БМТ, поскольку все величины, используемые в (3), являются элементами алгебры Березина. Переход от них к действительным числам может быть осушествлен с помошью функции плотности обычным образом [11]. С физической точки зрения второе слагаемое в первом уравнении есть сила Лоренца, а третье слагаемое - сила Кельвина [12], [13], обусловленная взаимодействием спина с градиентом электромагнитного поля.

В рамках этой модели в работе [10] были получены уравнения движения и траектории движения электрона, а также эволюции его спина для случая кулоновского поля. 


\section{3. ВЫЧИСЛЕНИЕ УГЛА РАССЕЯНИЯ}

В случае движения электрона в кулоновском поле массивного ядра $\varphi=Z e / r$, где $Z e-$ заряд ядра, можно получить компактное аналитическое выражение для угла рассеяния. Для этого воспользуемся полученным в [10] аналитическим выражением для траектории. Уравнение траектории в полярных координатах для притягиваюшего центра имеет вид

$$
\begin{gathered}
r\left(\alpha_{0}\right)=\frac{P}{1+D_{0} \cos \omega \alpha_{0}+\Lambda H\left(\cos \omega \alpha_{0}+1\right)}, \quad \gamma\left(\alpha_{0}\right)=\frac{\pi}{2}+\Lambda A \cos \alpha_{0}, \\
\alpha_{0}=\frac{\alpha}{1-\Lambda B},
\end{gathered}
$$

где $\alpha$ - азимутальный угол, $\gamma$ - полярный угол, $r$ - радиус-вектор, $\omega=\sqrt{1-\varrho^{2}}, \varrho=$ $q Q /(c K)(\varrho<1)$

$$
\begin{gathered}
P=\frac{K^{2} c^{2}-Z^{2} e^{4}}{Z e^{2} E}, \quad H=\frac{2 m Z e^{2} c^{2}}{K^{2} c^{2}-Z^{2} e^{4}}, \quad B=\frac{m Z e^{2} c}{K \sqrt{K^{2} c^{2}-Z^{2} e^{4}}}, \\
D_{0}=\frac{1}{\varrho} \sqrt{1-\frac{m^{2} c^{4}}{E^{2}}\left(1-\varrho^{2}\right)},
\end{gathered}
$$

$D_{0}, A$ - константы интегрирования, $K$ - компонента 4-импульса, отвечающая углу $\alpha$, $E$ - энергия налетаюшего электрона $\left(E>m c^{2}\right), \Lambda$ - интеграл движения, связанный со спином электрона, а именно компонента $-S^{01}$ 4-тензора спина $\left(\Lambda^{2}=0\right), m$ - масса электрона.

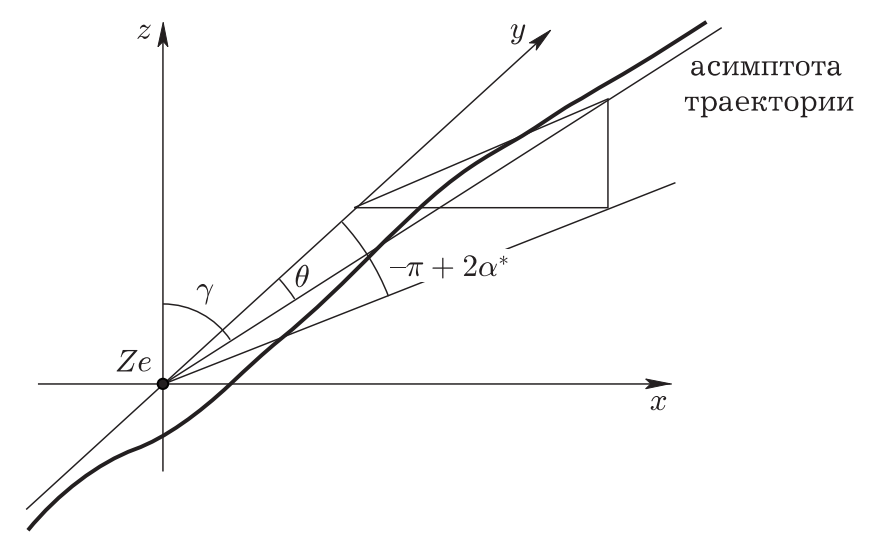

РИс. 1. Определение угла рассеяния для пространственной траектории электрона.

Зная уравнение траектории, легко получить угол $\alpha_{0}^{*}$, при котором $r$ стремится к бесконечности:

$$
\alpha_{0}^{*}=\frac{\pi}{\omega}-\frac{1}{\omega} \arccos \left(\frac{\Lambda H+1}{D_{0}+\Lambda H}\right) .
$$




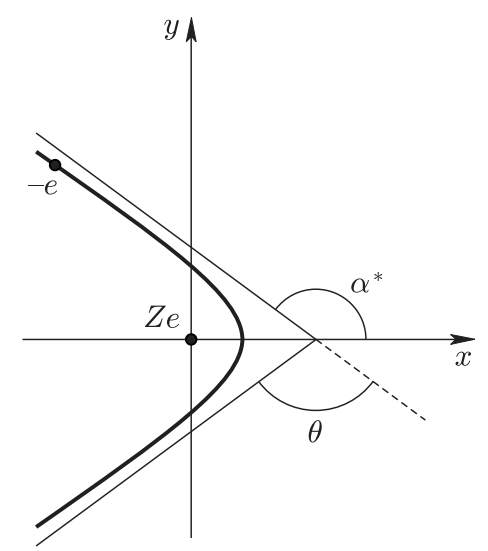

Рис. 2. Проекция траектории электрона вблизи притягивающего центра на плоскость $x O y$.

Угол $\alpha^{*}$ находится согласно (5), а угол рассеяния (см. рис. 1,2$)$ по формуле

$$
\theta=\arccos \left\{\sin \left(\gamma\left(\alpha^{*}\right)\right) \cos \left(-\pi+2 \alpha^{*}\right)\right\}
$$

С учетом нильпотентности $\Lambda$

$$
\begin{gathered}
\cos \alpha=\cos \left(\alpha_{0}-\Lambda B \alpha_{0}\right)=\cos \alpha_{0}+\Lambda B \alpha_{0} \sin \alpha_{0}, \\
\gamma(\alpha)=\frac{\pi}{2}+\Lambda A \cos \alpha=\frac{\pi}{2}+\Lambda A \cos \alpha_{0}=\gamma\left(\alpha_{0}\right) \\
\sin \gamma=\sin \left(\frac{\pi}{2}+\Lambda A \cos \alpha_{0}\right)=\sin \frac{\pi}{2}+\Lambda A \cos \alpha_{0} \cos \frac{\pi}{2}=1
\end{gathered}
$$

получим

$$
\theta=-\pi+2 \alpha^{*}
$$

Окончательное выражение для угла рассеяния в релятивистском случае при наличии спина частицы в рамках псевдоклассической механики (притягивающий центр) имеет вид

$$
\theta=-\pi+\frac{2 \pi}{\omega}-\frac{2}{\omega} \arccos \left(\frac{\Lambda H+1}{D_{0}+\Lambda H}\right)-\Lambda B\left\{\frac{2 \pi}{\omega}-\frac{2}{\omega} \arccos \left(\frac{\Lambda H+1}{D_{0}+\Lambda H}\right)\right\}
$$

Асимметрия угла рассеяния при этом учитьвается знаком величины $\Lambda$, которая может принимать как отрицательные, так и положительные значения.

С помошью аналогичных рассуждений для отталкивающего центра, уравнение траектории для которого имеет вид

$$
\begin{gathered}
r\left(\alpha_{0}\right)=\frac{P}{-1+D_{0} \cos \omega \alpha_{0}+\Lambda H\left(\cos \omega \alpha_{0}-1\right)}, \quad \gamma\left(\alpha_{0}\right)=\frac{\pi}{2}+\Lambda A \cos \alpha_{0}, \\
\alpha_{0}=\frac{\alpha}{1+\Lambda B},
\end{gathered}
$$


получим следующее выражение для угла рассеяния (рис. 3):

$$
\theta=\pi-\frac{2}{\omega} \arccos \left(\frac{\Lambda H+1}{D_{0}+\Lambda H}\right)-\frac{2 \Lambda B}{\omega} \arccos \left(\frac{\Lambda H+1}{D_{0}+\Lambda H}\right)
$$

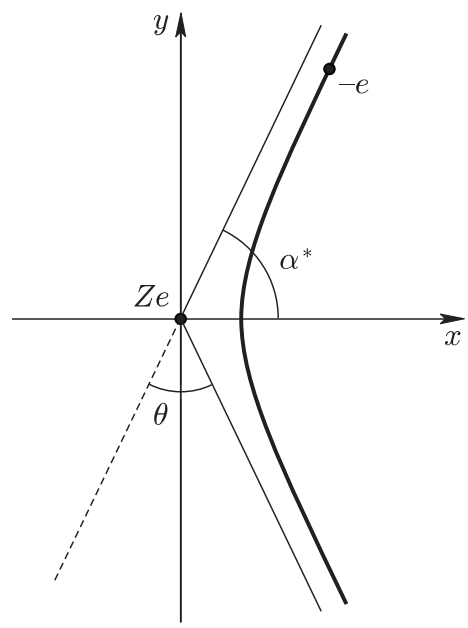

Рис. 3. Проекция траектории электрона вблизи отталкивающего центра на плоскость $x O y$.

Полученные выражения (6), (7) при $\Lambda=0$ совпадают с выражениями для угла рассеяния в релятивистском бесспиновом случае [14].

\section{4. ВЫЧИСЛЕНИЕ СЕЧЕНИЯ РАССЕЯНИЯ}

Чтобы получить явную формулу сечения рассеяния, необходимо из формулы (например, для (7))

$$
\begin{aligned}
\theta= & \pi-\frac{2}{\sqrt{1-Z^{2} e^{4} /\left(r_{0}^{2} c^{2} p^{2}\right)}}\left(1+\frac{c e^{2} m Z \Lambda}{r_{0} p \sqrt{r_{0}^{2} c^{2} p^{2}-Z^{2} e^{4}}}\right) \times \\
& \times \arccos \left(\frac{1+2 c^{2} e^{2} m Z \Lambda /\left(r_{0}^{2} c^{2} p^{2}-Z^{2} e^{4}\right)}{r_{0} v_{0} \sqrt{p^{2}+Z^{2} e^{4} /\left(r_{0}^{2} v_{0}^{2}\right)} /\left(Z e^{2}\right)+2 c^{2} e^{2} m Z \Lambda /\left(r_{0}^{2} c^{2} p^{2}-Z^{2} e^{4}\right)}\right)
\end{aligned}
$$

определить зависимость прицельного параметра $r_{0}$ от $\theta$ (здесь $v_{0}$ - скорость, а $p$ - импульс налетающего электрона на бесконечности). Сделать это в аналитическом виде невозможно (как это не сделано и в бесспиновом случае), но можно воспользоваться численными методами (рассмотрение численных решений выходит за рамки данной статьи). 
Исследуем данные формулы при больших прицельных параметрах, которым, как известно, отвечают малые углы рассеяния. В этом случае, например, формула (6) примет вид

$$
\theta \approx \frac{2 Z e^{2}}{v_{0} r_{0} p}+\frac{Z e^{2} \Lambda \pi}{m c v_{0} p r_{0}^{2}}
$$

что позволит найти интересующую нас зависимость $r_{0}$ от $\theta$.

Заметим, что к такому же результату можно прийти в процессе обшеизвестных [15] элементарных рассуждений. Рассмотрим движение электрона в кулоновском поле массивного ядра $\varphi=Z e / r$ при большом прицельном параметре (выберем ось $y$ в направлении первоначального движения электрона). В системе покоя ядра, считая траекторию электрона прямой (угол рассеяния мал) и лежашей в плоскости $x O y$, найдем импульс, переданный ядру:

$$
p_{x}=\int_{-\infty}^{\infty}\left[Z e E_{x}(t)+\left(F_{S E}\right)_{x}(t)\right] d t
$$

где $Z e E_{x}(t)$ и $\left(F_{S E}\right)_{x}(t)-x$-компоненты силы Кулона и силы Кельвина, соответственно, действуюшие на ядро со стороны налетаюшего электрона в момент времени $t$. Модуль силы $\mathbf{F}_{S E}$, как было ранее показано в работе [10], равен

$$
F_{S E}=\frac{1}{m c} \frac{2 Z e^{2} \Lambda}{r^{3}}
$$

Таким образом,

$$
\begin{aligned}
E_{x} & =\frac{e r_{0}}{\sqrt{1-(v / c)^{2}}\left[r_{0}^{2}+(v t)^{2} /\left(1-(v / c)^{2}\right)\right]^{3 / 2}} \\
\left(F_{S E}\right)_{x} & =\frac{2 Z e^{2} \Lambda r_{0}}{m c \sqrt{1-(v / c)^{2}}\left[r_{0}^{2}+(v t)^{2} /\left(1-(v / c)^{2}\right)\right]^{2}}
\end{aligned}
$$

где $v$ - скорость электрона в момент времени $t$.

Подставляя (10) и (11) в (9) и интегрируя, получим

$$
p_{x}=\frac{2 Z e^{2}}{v_{0} r_{0}}+\frac{Z e^{2} \Lambda \pi}{m c v_{0} r_{0}^{2}} .
$$

По закону сохранения импульса $x$-компонента импульса налетающей частицы после столкновения будет отличаться от величины (12) только знаком, поэтому угол рассеяния равен

$$
\theta \approx \frac{\left|p_{x}\right|}{p}
$$

что совпадает с (8). 
Таким образом, дифференциальное сечение рассеяния для малых углов (с точностью до $\theta$ во втором множителе) имеет вид

$$
\frac{d \sigma}{d \Omega}=\frac{1}{\theta} r_{0}(\theta) \frac{d r_{0}(\theta)}{d \theta}=\left(\frac{2 Z e^{2}}{v_{0} p}\right)^{2} \frac{1}{\theta^{4}}\left(1+\frac{\pi v_{0}^{2} \Lambda}{4 Z e^{2} c} \theta\right)
$$

Первое слагаемое есть не что иное, как резерфордовское сечение рассеяния, а второе представляет собой поправку к сечению, обусловленную спином электрона.

Согласно правилам перехода от грассмановых величин теории к обычным числам [11] тензору спина при усреднении сопоставляется следуюшее численное выражение:

$$
\left\langle S^{\mu \nu}\right\rangle=u_{\alpha} S_{\beta} \varepsilon^{\alpha \beta \mu \nu}
$$

где $S_{\beta}$ - классический аналог вектора Паули-Любаньского, т.е. числовой 4-спин, а $u_{\alpha}-$ 4-скорость электрона.

Следовательно, величина $\Lambda$, входящая в сечение, должна иметь вид (движение в данном приближении плоское)

$$
\Lambda=\left\langle i \theta^{0} \theta^{1}\right\rangle=-\left\langle S^{01}\right\rangle=S_{3} u_{2},
$$

где $S_{3}$ - проекция вектора спина на ось $O z, u_{2}$ - компонента 4-скорости по угловой координате $\alpha$.

Для сопоставления полученного выражения (13) с формулой Мотта [5]

$$
\frac{d \sigma}{d \Omega}=\left(\frac{Z e^{2}}{2 v_{0} p}\right)^{2} \frac{1}{\sin ^{4}(\theta / 2)}\left(1-\frac{v_{0}^{2}}{c^{2}} \sin ^{2} \frac{\theta}{2} \pm \frac{\pi Z e^{2} v_{0}}{\hbar c^{2}} \sin \frac{\theta}{2}\left(1-\sin \frac{\theta}{2}\right)\right)
$$

поступим следующим образом. Формула (14) получена при выборе определенной поляризации налетающих электронов. В псевдоклассической модели это означает фиксацию проекции спина на выделенное направление. Как показано в работе [11], матрица плотности, возникаюшая после квантования модели Ди Векиа-Равндела, будет положительно-полуопределенной, если только пространственные компоненты вектора спина $\left|S_{k}\right| \leqslant \hbar / 2$. Но если налетаюший электрон в некоторый момент времени имеет фиксированное значение проекции спина $\left|S_{3}\right|=\hbar / 2$, то значение величины $u_{2}$ в этот момент не может быть выбрано произвольно, так как оно представляет собой некоторую функцию от параметров $Z, v_{0}, S_{3}$, определяющих траекторию электрона. Значение $u_{2}$ удобно записать в форме

$$
u_{2}=\frac{\Lambda_{0} Z e^{2}}{\hbar c}
$$

где $\Lambda_{0}$ - некоторая безразмерная числовая константа. Такая форма естественна, если исходить из выражения для скорости в теории Бора [15]

$$
v_{n}=\frac{Z e^{2}}{\hbar} \frac{1}{n}, \quad n=1,2,3, \ldots
$$


Таким образом, величина $\Lambda$, определяющая вклад спина в сечение рассеяния, запишется в виде

$$
\Lambda= \pm\left|S_{3}\right| u_{2}= \pm \frac{\Lambda_{0} Z e^{2}}{2 c} .
$$

Подставляя полученное выражение в (13), имеем

$$
\frac{d \sigma}{d \Omega}=\left(\frac{2 Z e^{2}}{v_{0} p}\right)^{2} \frac{1}{\theta^{4}}\left(1 \pm \frac{\pi Z e^{2} v_{0}}{2 \hbar c^{2}} \frac{\Lambda_{0}}{4} \theta\right)
$$

Сравнивая полученное выражение с формулой Мотта (14), убеждаемся, что они совпадают для малых углов при значении $\Lambda_{0}=4$. Это значение и нужно использовать при численном расчете сечения рассеяния на произвольные углы по формулам (6), (7).

\section{5. ЗАКЛЮЧЕНИЕ}

Спин электрона имеет квантовую природу, что наводит на мысль о невозможности какого-либо классического его описания. В свое время это составило основу критики гипотезы о спине, как свойстве классического врашающегося волчка. Наиболее развитая из классических теорий спина - релятивистская модель точечного волчка Френкеля [16] - давала поправку к расчету спин-орбитального взаимодействия (поправку Томаса-Френкеля), в два раза большую, чем экспериментальные значения.

После создания квантовой релятивистской модели спина на основе уравнения Дирака модель Френкеля была отброшена. Однако уравнения БМТ возникали и в теории Френкеля. Это связано с тем обстоятельством, что уравнение эволюции спина БМТ имеет классический характер - оно не содержит явно постоянной Планка. Эти уравнения можно рассматривать как классическое приближение общего уравнения эволюции спина в представлении Гейзенберга [16]. Переход к уравнениям БМТ фактически означает пренебрежение эффектами "квантового дрожания" (Zitterbewegung) электрона.

Псевдоклассическая модель электрона, рассмотренная в данной статье, лишена недостатков классических моделей. Она дает правильное гиромагнитное отношение, при квантовании переходит в модель Дирака, "квантовому дрожанию" в ней отвечают нечетные грассмановы переменные, которые исчезают при усреднении [9], [11]. Поэтому все, что отличает предсказание этой модели от квантовой модели Дирака, представляет собой “чисто квантовый” вклад природы спина. Как всякая классическая модель, она содержит множество подробностей, отсутствуюших в квантовой модели (траектории, прицельные параметры и т.п.).

Сопоставляя классические решения с квантовыми, можно получить ограничения на параметры модели. Так, сравнение с расчетами Мотта дает ограничение на величину азимутальной составляющей скорости

$$
u_{2} \geqslant \frac{4 Z e^{2}}{\hbar c} .
$$


При фиксации величины проекции спина $\left|S_{3}\right|=\hbar / 2$ это неравенство преврашается в некоторое условие квантования, аналогичное условию Бора-Зоммерфельда в старой квантовой теории.

В качестве дальнейшего развития предлагаемого подхода можно исследовать случай финитных траекторий электрона в кулоновском поле, т.е. построить псевдоклассическую модель "атома по Бору со спином". Кроме обычных условий квантования из рассмотрения спин-орбитальных поправок в ней должны возникать условия квантования на компоненты спина, аналогичные (15).

\section{Список литературы}

[1] Л. Д. Ландау, Е. М. Лифииц. Механика. М.: Наука, 1988.

[2] Л. Д. Ландау, Е. М. Лифииц. Теория поля. М.: Наука, 1988.

[3] Л. Д. Ландау, Е. М. Лифииц. Квантовая механика. М.: Наука, 1988.

[4] В. М. Галицкий, Б. М. Карнаков, В.И. Коган. Задачи по квантовой механике. М.: Наука, 1992.

[5] Н. Мотm, Г. Месси. Теория атомных столкновений. М.: Мир, 1969.

[6] F. A. Berezin, M.S. Marinov. Ann. Phys. 1977. V. 104. P. 336.

[7] Ю.Р. Мусин, В.В. Чередов. Введение в суперсимметричную механику. Депонировано в ВИНИТИ. № 8713-В88. М.: ВИНИТИ, 1988.

[8] F. Ravndal. Phys. Rev. D. 1980. V. 21. P. 2832.

[9] P. G. O. Freund. Introduction to Supersymmetry. Berlin: Springer, 1986.

[10] Ю.Р. Мусин, В. И. Козориз. ТМФ. 2000. Т. 123. № 1. С. 75.

[11] Ю. Р. Мусин. Изв. вузов. Физика. 1991. Т. 7. С. 5.

[12] H. Rumpf. Gen. Relativity Gravitation. 1982. V. 14. № 9. Р. 773.

[13] С. Р. де Гроот, Л. Г. Сатторп. Электродинамика. М.: Наука, 1982.

[14] H. Frommert. Int. J. Theor. Phys. 1996. V. 35. № 12. P. 2631.

[15] Д. Бом. Квантовая теория. М.: Наука, 1965.

[16] И. М. Тернов. Введение в физику спина релятивистских частиц. М.: Изд-во МГУ, 1997.

Поступила в редакцию 21.III.2003 г. 